Pacific Journal of Mathematic 


\section{ON THE RATE OF DECAY OF SOLUTIONS OF PARABOLIC DIFFERENTIAL EQUATIONS}

\section{J. K. ODDSON}

In this paper upper and lower bounds are determined for the rate of growth or decay of solutions of parabolic equations for indefinitely increasing time. These bounds are obtained from comparison functions which are constructed systematically with the aid of elliptic extremal operators and in many cases are the best possible for the class of problems which we consider.

The behavior of solutions of parabolic equations for indefinitely increasing time has been investigated by several authors in recent years. We refer in particular to the papers [2], [3] and [9] and the survey article [7]. Most of these articles have been concerned with establishing the approach of solutions to a steady state condition under various assumptions on the coefficients. In the present paper we determine upper and lower bounds for the rate at which solutions decay (or possibly grow) in a half cylinder as $t \rightarrow \infty$. These results, presented in Theorems 1 and 2, are obtained for a class of parabolic equations, assuming only uniform parabolicity and boundedness of the coefficients. The main tool is the maximum principle and the use of comparison functions constructed with the aid of elliptic extremal operators $[15,16]$. By this device the estimates which we obtain are often the best possible for the class of operators which we consider.

2. Notations and basic hypotheses. Let $\Omega$ be a bounded, open, connected subset of $E^{n}(n \geqq 1)$ with boundary $\Gamma$ and closure $\bar{\Omega}=\Omega \cup \Gamma$. Let $D$ denote the $(n+1)$-dimensional half cylinder $D=\Omega \times(0, \infty)$.

In this paper we shall be concerned with the linear differential operator

$$
\begin{aligned}
L-k \frac{\partial}{\partial t} \equiv \sum_{i, j=1}^{n} a_{i j}(x, t) \frac{\partial^{2}}{\partial x_{i} \partial x_{j}} & +\sum_{i=1}^{n} b_{i}(x, t) \frac{\partial}{\partial x_{i}} \\
& +c(x, t)-k(x, t) \frac{\partial}{\partial t}
\end{aligned}
$$

with real valued coefficients defined for all $(x, t) \equiv\left(x_{1}, \cdots, x_{n}, t\right) \in D$ and satisfying there the following hypothesis $I$ :

(1) $\sum_{i=1}^{n} a_{i i}(x, t) \equiv 1$ for all $(x, t) \in D$;

(2) There exists a constant $\alpha, 0<\alpha \leqq 1 / n$, such that $\sum_{i, j=1}^{n} a_{i j}(x, t) \xi_{i} \xi_{j} \geqq \alpha \sum_{i=1}^{n} \xi_{i}^{2}$ for all $(x, t) \in D$ and all real $n$-vectors $\left(\xi_{1}, \cdots, \xi_{n}\right)$.

( 3 ) $b_{1}(x, t), \cdots, b_{n}(x, t), c(x, t)$ are bounded in $D$; 
(4) There exist constants $k_{0}, k_{1}$ such that $0<k_{0} \leqq k(x, t) \leqq k_{1}$ in $D$.

We remark that any uniformly parabolic operator of the form $\sum_{i, j=1}^{n} A_{i j}\left(\partial^{2} / \partial x_{i} \partial x_{j}\right)+\sum_{i=1}^{n} B_{i}\left(\partial / \partial x_{i}\right)+c-(\partial / \partial t)$ with bounded coefficients can be normalized to the form (2.1) by dividing by $\sum_{i=1}^{n} A_{i i}$.

Note that the conditions $I(1)$ and $I(2)$ imply that $\sum_{i, j=1}^{n} a_{i j} \xi_{i} \xi_{j} \leqq$ $[1-(n-1) \alpha] \sum_{i=1}^{n} \xi_{i}^{2}$ in $D$ for all real $n$-vectors $\left(\xi_{1}, \cdots, \xi_{n}\right)$ and that in the case $\alpha=1 / n$ the principal part of the operator $L$ reduces to $(1 / n) \Delta$, where $\Delta$ denotes the $n$-dimensional Laplacian.

It is well known $[4-7,10,14,17-18]$ that under the hypotheses $I$ the operator (2.1) enjoys the maximum and boundary point principles. Since we wish to permit possible discontinuities in our solutions $u(x, t)$ on the initial surface $\bar{\Omega} \times\{0\}$ we state these principles in the following form: Suppose that $u(x, t)$ is a real valued function, differentiable with respect to $t$ and twice differentiable with respect to $x$ in $D$, continuous on $\bar{\Omega} \times(0, \infty)$, and satisfying $L u-k(\partial u / \partial t) \geqq 0$ in $D$. Suppose that $u \leqq 0$ on $\Gamma \times(0, \infty)$ and that $\varlimsup_{(x, t) \rightarrow \bar{a} \times\{0\}} u(x, t) \leqq 0$. Then $u(x, t) \leqq 0$ in $\bar{\Omega} \times(0, \infty)$. If $u\left(x^{0}, t_{0}\right)=0$ for some point $\left(x^{0}, t_{0}\right) \in D$ then $u(x, t) \equiv 0$ for all $x \in \bar{\Omega}, 0<t \leqq t_{0}$. If $u\left(x^{0}, t_{0}\right)=0$ for some point $\left(x^{0}, t_{0}\right) \in \Gamma \times(0, \infty)$ and if $\Gamma$ has the inner sphere property ${ }^{1}$ at $x^{0}$ then either $u(x, t) \equiv 0$ for all $x \in \bar{\Omega}, 0<t \leqq t_{0}$ or else there exists a positive constant $m$ such that $u\left(x, t_{0}\right) \leqq-m\left\|x-x^{0}\right\|$ for all $x$ within the prescribed sphere along a fixed line segment from $x^{0}$ and sufficiently close to $x^{0}$. Here we define

$$
\|x\|=\left(\sum_{i=1}^{n} x_{i}^{2}\right)^{1 / 2}
$$

With the aid of the maximum principle we establish the following

LEMMA 1. Suppose that hypotheses I hold. Let $u(x, t)$ be a real valued function, differentiable with respect to t and twice differentiable with respect to $x$ in $D$, continuous on $\bar{\Omega} \times(0, \infty)$, and satisfying $L u-k(\partial u / \partial t) \geqq 0$ in $D$. Suppose that $u \leqq 0$ on $\Gamma \times(0, \infty)$ and that $\varlimsup_{(x, t) \rightarrow \bar{\Omega} \times\{0\}} u(x, t) \leqq M$. If $u\left(x^{0}, t_{0}\right)=0$ for some point $\left(x^{0}, t_{0}\right) \in \Gamma \times(0, \infty)$ and if $\Gamma$ has the exterior sphere property ${ }^{1}$ at $x^{0}$ then there exists a positive constant $C$ such that $u\left(x, t_{0}\right) \leqq C\left\|x-x^{0}\right\|$ for all $x$ in $\bar{\Omega}$ and sufficiently close to $x^{0}$.

Proof. Assume first that $c(x, t) \leqq 0$. Without loss of generality we may suppose that the axes have been translated so that the exterior sphere prescribed at $x^{0}$ is centered at the origin and has radius $r_{0}=\left\|x^{0}\right\|$. Define $r=\|x\|$ and $\rho^{2}=\|x\|^{2}+\left(t-t_{0}\right)^{2}$. Consider now the $(n+1)$-dimensional sphere $B=\left\{(x, t): \rho^{2}<r_{0}^{2}\right\}$, which is exterior to

${ }^{1} \Gamma$ has the inner (exterior) sphere property at $x^{0}$ if there exists an open ball $B$ contained in $\Omega$ (the complement of $\bar{\Omega}$ ) such that $\bar{B} \cap \Gamma=\left\{x^{0}\right\}$. 
$D$ at the point $\left(x^{0}, t_{0}\right)$, and the concentric sphere $B^{\prime}=\left\{(x, t): \rho^{2}<\left(r_{0}+\varepsilon\right)^{2}\right\}$ where $\varepsilon>0$ is chosen to be suitably small.

Define $Q^{\prime}$ to be the open subset of $D$ enclosed by $B^{\prime}$ for $0<t<t_{0}, \Gamma^{\prime}$ to be the closure of $B^{\prime} \cap\left\{\Gamma \times\left(0, t_{0}\right)\right\}$, and $S^{\prime}$ to be the subset of the surface of $B^{\prime}$ contained in $D$ for $0<t \leqq t_{0}$.

In the closure of $\Omega^{\prime}$ consider the function $h(x, t)=e^{-\hat{\delta} r_{0}^{2}}-e^{-\delta \rho^{2}}$, where $\delta$ is a constant to be chosen subsequently. Note that $h>0$ in the closure of $\Omega^{\prime}$ with the exception of the point $\left(x^{0}, t_{0}\right)$ where we have $h\left(x^{0}, t_{0}\right)=0$.

In $Q^{\prime}$ we have

$$
\begin{aligned}
L h-k \frac{\partial h}{\partial t} & =c h+2 \delta e^{-\delta \rho^{2}}\left\{1-2 \delta \sum_{i, j=1}^{n} a_{i j} x_{i} x_{j}+\sum_{i=1}^{n} b_{i} x_{i}-k\left(t-t_{0}\right)\right\} \\
& \leqq 2 \delta e^{-\delta \rho^{2}}\left\{A-2 \delta \alpha\left\|x^{0}\right\|^{2}\right\} \\
& <0 \text { if } \delta \text { is chosen to be sufficiently large. }
\end{aligned}
$$

It is an easy consequence of the weak form of the maximum principle mentioned earlier that $u(x, t) \leqq \max \{0, M\}$ in $D$ and hence there exists a positive constant $C$ such that $u \leqq C h$ for $(x, t) \in S^{\prime}$.

If we now define $w=u-C h$ we have $L w-k(\partial w / \partial t)>0$ in $\Omega^{\prime}$ while $w \leqq 0$ in $S^{\prime} \cup \Gamma^{\prime}$. It follows from the weak maximum principle that $w \leqq 0$ in the closure of $\Omega^{\prime}$. In particular we have $u\left(x, t_{0}\right) \leqq$ $C\left\{e^{-\delta r_{0}^{2}}-e^{\delta r^{2}}\right\}$ for $x \in \bar{\Omega}, r \leqq r_{0}+\varepsilon$, which immediately implies the result of the lemma. Note that the constant $C$ is independent of $t_{0}$ in this case.

For the more general case when $c(x, t) \leqq \gamma, \gamma>0$, we apply the above result to the function $v(x, t)=e^{-\gamma t / k_{0}} u(x, \mathrm{t})$ which satisfies the inequality $L v-k(\partial v / \partial t) \geqq 0$ in $D$ for an operator of type (2.1) with $c(x, t) \leqq 0$. We obtain $v\left(x, t_{0}\right) \leqq C\left\{e^{-\delta r_{0}^{2}}-e^{-\hat{o} r^{2}}\right\}$ and thus $u\left(x, t_{0}\right) \leqq$ $C e^{\gamma t_{0} / k_{0}}\left\{e^{-\delta r^{2} 0}-e^{-\delta r^{2}}\right\}$.

3. Extremal operators and the class $\mathscr{L}_{\alpha}$. Let us denote by $\mathscr{L}_{\alpha}$ the class of uniformly elliptic operators of the form

$$
L^{\prime}=\sum_{i, j=1}^{n} a_{i j}(x, t) \frac{\partial^{2}}{\partial x_{i} \partial x_{j}}
$$

with real valued coefficients defined in $D$ and satisfying there the hypotheses $I(1)$ and $I(2)$. In recent papers $[15,16]$ Pucci has introduced the maximizing and minimizing operators, relative to the class $\mathscr{L}_{\alpha}$, defined respectively by

$$
\begin{aligned}
& M_{\alpha}[u](x, t)=\sup _{L^{\prime} \in \mathscr{S}_{\alpha}}\left\{L^{\prime} u(x, t)\right\} \\
& m_{\alpha}[u](x, t)=\inf _{L^{\prime} \in \mathscr{L}_{\alpha}}\left\{L^{\prime} u(x, t)\right\}
\end{aligned}
$$

for any given function $u(x, t)$ twice differentiable with respect to $x$ in $D$. 
He has shown that these operators have the nonlinear representations

$$
\begin{aligned}
& M_{\alpha}[u]=\alpha \Delta u+(1-n \alpha) C_{n}(u) \\
& m_{\alpha}[u]=\alpha \Delta u+(1-n \alpha) C_{1}(u)
\end{aligned}
$$

where $\Delta$ denotes the $n$-dimensional Laplacian and $C_{1}(u) \leqq C_{2}(u) \leqq \cdots \leqq$ $C_{n}(u)$ denote the ordered eigenvalues of the $n \times n$ Hessian matrix $\left(u_{i j}(x, t)\right)$.

For functions $u(x, t)=R(r, t)$ which depend only upon $t$ and the distance $r \equiv\|x\|$ from a fixed point (taken to be the origin) the representations (3.2) reduce to the simpler forms

$$
\begin{aligned}
& M_{\alpha}[u]=h_{1} \frac{\partial^{2} R}{\partial r^{2}}+\frac{\left(1-h_{1}\right)}{r} \frac{\partial R}{\partial r} \\
& m_{\alpha}[u]=h_{2} \frac{\partial^{2} R}{\partial r^{2}}+\frac{\left(1-h_{2}\right)}{r} \frac{\partial R}{\partial r}
\end{aligned}
$$

where $h_{1}=\alpha$ and $h_{2}=1-(n-1) \alpha$ if $\left(\partial^{2} R / \partial r^{2}\right)<(1 / r)(\partial R / \partial r)$ while $h_{1}=1-(n-1) \alpha$ and $h_{2}=\alpha$ if $\left(\partial^{2} R / \partial r^{2}\right) \geqq(1 / r)(\partial R / \partial r)$.

From the definitions it is clear that for any function $u(x, t)$, twice differentiable with respect to $x$ in $D$, and any $L^{\prime} \in \mathscr{L}_{\alpha}$ we have the inequality $m_{\alpha}[u](x, t) \leqq L^{\prime} u(x, t) \leqq M_{\alpha}[u](x, t)$ for each $(x, t) \in D$. However, it may also be shown that there exist operators $L_{1}^{\prime}, L_{2}^{\prime} \in L_{\alpha}$ (with coefficients determined by $u$ ) such that $L_{1}^{\prime} u(x, t)=m_{\alpha}[u](x, t)$ and $L_{2}^{\prime} u(x, t)=M_{\alpha}[u](x, t)$ for all $(x, t) \in D$, thereby justifying the terminology.

We refer to the papers $[8,11-13,16]$ for a more detailed development of the theory of these extremal operators and their applications. Their significance for our purposes in this paper rests on the fact that they provide a systematic method for determining comparison functions for our parabolic operator (2.1). The construction of these functions is based upon the following lemma. Here $J_{\mu}$ denotes the Bessel function of the first kind of order $\mu$ in the notation of [1], and $r=\|x\|=\left(\sum_{i=1}^{n} x_{i}^{2}\right)^{1 / 2}$.

Lemma 2. Let $\alpha, \beta, \gamma, \kappa$ be constants with $0<\alpha \leqq 1 / n$, and $\beta>-1$. If we set

$$
\mu=\frac{\beta+1}{2[1-(n-1) \alpha]}-1 \text { and } \lambda=\frac{\xi_{0}^{2}[1-(n-1) \alpha]-\gamma r_{0}^{2}}{\kappa r_{0}^{2}},
$$

where $\xi_{0}$ is the first positive zero of $J_{\mu}(\xi)$, then the function $r^{-\mu} J_{\mu}\left\{\left(\xi_{0} r / r_{0}\right)\right\} e^{-\lambda t}$ is a solution of the equation

$$
M_{\alpha}[u]+\frac{\beta}{r} \frac{\partial u}{\partial r}+\gamma u-\kappa \frac{\partial u}{\partial t}=0
$$


in the $(n+1)$-dimensional cylinder $Q=\left\{(x, t): r<r_{0}, t>0\right\}$ which is positive in $Q$, decreasing with respect to $r$, and zero on the cylindrical surface $r=r_{0}$. If, instead, we set

$$
\mu=\frac{(\beta+1)}{2 \alpha}-1 \text { and } \gamma=\frac{\alpha \xi_{0}^{2}-\gamma r_{0}^{2}}{\kappa r_{0}^{2}}
$$

then this function is a solution of the equation

$$
m_{\alpha}[u]+\frac{\beta}{r} \frac{\partial u}{\partial r}+\gamma u-\kappa \frac{\partial u}{\partial t}=0
$$

in $Q$ which is positive in $Q$, decreasing with respect to $r$, and zero on the surface $r=r_{0}$.

4. The decay estimates. With the aid of the maximum principle and the previous lemmas we can now establish our main results on the decay rate of solutions associated with our parabolic operator (2.1).

To obtain an upper bound on the decay rate we suppose that the set $\Omega$ has diameter $2 r_{0}$ and is contained in the sphere of radius $r_{0}$ centered at the point $x^{0} \in E^{n}$. The set $D$ is then enclosed in the $(n+1)$ dimensional half cylinder $Q=\left\{(x, t):\left\|x-x^{0}\right\|<r_{0}, t>0\right\}$.

THEOREM 1. Suppose that hypotheses I hold and that

$$
\inf _{D} \sum_{i=1}^{n} b_{i}\left(x_{i}-x_{i}^{0}\right) \equiv \beta>-1 \text { and } \sup _{D} c(x, t)=\gamma \text {. }
$$

Define

$$
\mu=\frac{(\beta+1)}{2[1-(n-1) \alpha]}-1 \text { and } \lambda=\frac{\xi_{0}^{2}[1-(n-1) \alpha]-\gamma r_{0}^{2}}{\kappa r_{0}^{2}}
$$

where $\xi_{0}$ is the first positive zero of $J_{\mu}(\xi)$ and $\kappa=k_{1}$ if $\xi_{0}^{2}[1-(n-1) \alpha] \geqq \gamma r_{0}^{2}$, while $\kappa=k_{0}$ if $\xi_{0}^{2}[1-(n-1) \alpha]<\gamma r_{0}^{2}$.

Suppose that $u(x, t)$ is a real valued function, differentiable with respect to $t$ and twice differentiable with respect to $x$ in $D$, continuous on $\bar{\Omega} \times(0, \infty)$, and satisfying $L u-k(\partial u / \partial t) \geqq 0$ in $D$. If $u(x, t) \leqq 0$ $\overline{\text { on }} \Gamma \times(0, \infty)$ and $\overline{\lim }_{(x, t) \rightarrow \bar{\Omega} \times\{0\}} u(x, t) \leqq M$ then there exists a positive constant $C$ such that $u(x, t) \leqq C \rho e^{-\lambda t}$ in $\bar{\Omega} \times(0, \infty)$ where $\rho$ denotes the distance from $x$ to the lateral surface of $Q$.

Proof. We need only consider the case $M>0$ for otherwise the result follows trivially from the weak maximum principle. Moreover without loss of generality we may suppose that the axes have been translated so that the axis of the cylinder $Q$ is the $t$-axis.

Using the definition of the maximizing operator, the assumptions 
of the theorem, and Lemma 2 we note that the function $V(r, t)=$ $r^{-\mu} J_{\mu}\left(\xi_{0} r / r_{0}\right) \mathrm{e}^{-\lambda t}$ satisfies the differential inequality

$$
\begin{aligned}
L V & -k \frac{\partial V}{\partial t} \leqq M_{\alpha}[V]+\frac{\left(\sum_{i=1}^{n} b_{i} x_{i}\right)}{r} \frac{\partial V}{\partial r}+c V-k \frac{\partial V}{\partial t} \\
& \leqq M_{\alpha}[V]+\frac{\beta}{r} \frac{\partial V}{\partial r}+\gamma V-\kappa \frac{\partial V}{\partial t}=0
\end{aligned}
$$

in $D$, where $\kappa$ is prescribed in the hypothesis of the theorem.

Let $0<\varepsilon<1$ be an arbitrary constant and denote by $D_{\varepsilon}$ the half cylinder $D_{\varepsilon}=\Omega \times(\varepsilon, \infty)$. Note that $\Gamma$ has the exterior sphere property at any point $x^{0} \in \Gamma$ such that $\left(x^{0}, \varepsilon\right)$ is a common boundary point of both $D_{\varepsilon}$ and $Q$. The result of Lemma 1 then implies that $\overline{\lim }_{x \rightarrow \Gamma}\{u(x, \varepsilon) / V(r, \varepsilon)\}$ is bounded above by a constant which may be chosen independent of $\varepsilon$. Moreover from the weak maximum principle we have $u(x, \varepsilon)$ bounded above, by $M$ if $\gamma \leqq 0$ and by $M e^{\gamma \varepsilon / k o}$ if $\gamma>0$, for all $x \in \Omega$. It follows that there exists a positive constant $a$, independent of $\varepsilon$, such that $u(x, \varepsilon)-a V(r, \varepsilon) \leqq 0$ for all $x \in \Omega$.

Combining these results we conclude that the function $w(x, t)=$ $u(x, t)-a V(r, t)$ satisfies the differential inequality $L w-k(\partial w / \partial t) \geqq 0$ in $D_{\varepsilon}$ and the inequality $w \leqq 0$ on $\Gamma \times[\varepsilon, \infty]$ and on $\Omega \times\{\varepsilon\}$. By the weak maximum principle we have $w \leqq 0$ in $\bar{\Omega} \times[\varepsilon, \infty)$, i.e., there exists a positive constant, $a$, such that

$$
u(x, t) \leqq a r^{-\mu} J_{\mu}\left(\frac{\xi_{0} r}{r_{0}}\right) e^{-\lambda t} \text { in } \bar{\Omega} \times[\varepsilon, \infty)
$$

and since $a$ is independent of $\varepsilon$ the same result holds in $\bar{\Omega} \times(0, \infty)$. This clearly implies the result of the theorem.

To obtain a lower bound for the decay rate we define $r_{1}$ to be the maximum of the radii of all open spheres contained in $\Omega$ and suppose one such largest sphere is $B$, centred at the point $x^{1} \in \Omega$. The set $D$ then contains the $(n+1)$-dimensional half cylinder $Q_{1}=B \times(0, \infty)=$ $\left\{(x, t):\left\|x-x^{1}\right\|<r_{1}, t>0\right\}$.

THEOREM 2. Suppose that hypotheses $I$ hold and that $\sup _{Q_{1}} \sum_{i=1}^{n} b_{i}\left(x_{i}-x_{i}^{1}\right)=\beta, \inf _{Q 1} c(x, t)=\gamma$. Define $\mu=\{(\beta+1) / 2 \alpha\}-1$ and $\lambda=\frac{\alpha \xi_{0}^{2}-\gamma r_{1}^{2}}{K r_{1}^{2}}$, where $\xi_{0}$ is the first positive zero of $J_{\mu}(\xi)$ and $K=k_{0}$ if $\alpha \xi_{0}^{2} \geqq \gamma r_{1}^{2}$ while $K=k_{1}$ if $\alpha \xi_{0}^{2}<\gamma r_{1}^{2}$.

Suppose that $u(x, t)$ is a real valued function, differentiable with respect to $t$ and twice differentiable with respect to $x$ in $D$, continuous on $\bar{\Omega} \times(0, \infty)$, and satisfying $L u-k(\partial u / \partial t) \leqq 0$ in $D$. If $u \geqq 0$ on $\Gamma \times(0, \infty)$, $\underline{\lim }_{(x, t) \rightarrow \Gamma \times\{0\}} u(x, t) \geqq 0$, and $\underline{\lim }_{(x, t) \rightarrow \Omega \times\{0\}} u(x, t)>0$ then there exists $a$ 
positive constant $m$ such that $u(x, t) \geqq m \rho e^{-\lambda t}$ in $B \times[\varepsilon, \infty)$ where $\rho$ denotes the distance from $x$ to the boundary of $B$ and $\varepsilon>0$ is arbitrarily small.

Proof. Again we may assume that the axes are translated so that the sphere $B$ is centred at the origin. Using the definition of the minimizing operator, the assumptions of the theorem, and Lemma 2 we note that the function $v(r, t)=r^{-\mu} J_{\mu}\left\{\left(\xi_{0} r / r_{1}\right)\right\} e^{-\lambda t}$ satisfies the differential inequality

$$
\begin{aligned}
L v & -k \frac{\partial v}{\partial t} \geqq m_{\alpha}[v]+\frac{\left(\sum_{i=1}^{n} b_{i} x_{i}\right)}{r} \frac{\partial v}{\partial r}+c v-k \frac{\partial v}{\partial t} \\
& \geqq m_{\alpha}[v]+\frac{\beta}{r} \frac{\partial v}{\partial r}+\gamma v-K \frac{\partial v}{\partial t}=0
\end{aligned}
$$

in $Q_{1}$, where $K$ is prescribed in the hypotheses of the theorem.

Let $0<\varepsilon<1$ be an arbitrary constant. Note that $u>0$ on $B \times\{\varepsilon\}$ by the strong minimum principle and the condition $\lim _{(x, t) \rightarrow \Omega \times\{0\}} u(x, t)>0$. Moreover the boundary point principle implies that $\varlimsup_{x \rightarrow \partial B}\{u(x, \varepsilon) / v(r, \varepsilon)\}$ is bounded below by a positive constant, since $\Gamma$ has the inner sphere property at any point common to $\Gamma$ and $\partial B$. It follows that there exists a positive constant $\delta$ such that $u(x, \varepsilon)-\delta v(r, \varepsilon) \geqq 0$ for all $x \in B$.

Combining these results we conclude that the function $w(x, t)=$ $u(x, t)-\delta v(r, t)$ satisfies the differential inequality $L w-k(\partial w / \partial t) \leqq 0$ in $B \times(\varepsilon, \infty)$ and the inequality $w \geqq 0$ on $\partial B \times[\varepsilon, \infty)$ and on $B \times[\varepsilon]$. By the weak minimum principle we have $w \geqq 0$ in $B \times[\varepsilon, \infty)$, i.e., there exists a positive constant $\delta$ such that $\left.u(x, t) \geqq \delta r^{-\mu} J_{\mu}\left\{\xi_{0} r / r_{1}\right)\right\} e^{-\lambda t}$ in $B \times[\varepsilon, \infty)$. The result of the theorem now follows directly.

5. Concluding remarks. We emphasize that for the class of operators of the form (2.1) with coefficients $b_{i} \equiv 0, i \equiv 1, \cdots, n$ and the case that $\Omega$ is a sphere our Theorems 1 and 2 cannot be improved. In fact from the discussion of the properties of the extremal operators it follows that there exists an operator in this class for which our comparison function $V(r, t)(v(r, t))$ is a solution in $\Omega \times(0, \infty)$.

Finally let us note that Theorems 1 and 2 may be used to obtain comparison theorems for more general nonhomogeneous parabolic equations by the device of subtracting off a suitable approximate solution of the equations.

\section{REFERENCES}

1. A. Erdelyi (editor), Higher Transcendental Functions, Vol. 2, McGraw-Hill, New York, New York, 1953. 
2. A. Friedman, Convergence of solutions of parabolic equations to a steady state, J. Math. Mech. 8 (1959), 57-76.

3. - Asymptotic behavior of solutions of parabolic equations, J. Math. Mech, 8 (1959), 387-392.

4. - Remarks on the maximum principle for parabolic equations and its applications, Pacific J. Math. 8 (1958), 201-211.

5. — A strong maximum principle for weakly subparabolic functions, Pacific J. Math. 11 (1961), 175-184.

6. - Partial differential equations of parabolic type, Prentice-Hall, Inc., Englewood Cliffs, N. J., 1964.

7. A. M. Il'in, A. S. Kalashnikov, and O. A. Oleinik, Second order linear equations of parabolic type, Uspekhi Math. Nauk SSSR 17 (1962), 3-146. (Translated in Russian Math. Surveys 17 (1962), 1-143.)

8. K. Miller, Non-existence of an a priori bound, etc., Ann. Mat. Pura Appl. 73 (1966), 11-16.

9. R. Narasimban, On the asymptotic stability of solutions of parabolic differential equations, J. Rat. Mech. Anal, 3 (1954), 303-319.

10. L. Nirenberg, A strong maximum principle for parabolic equations, Comm. Pure Appl. Math. 6 (1953), 167-177.

11. J.K. Oddson, Phragmen-Lindelof and comparison theorems for elliptic equations with mixed boundary conditions, Arch. Rat. Mech. Anal. 26 (1967), 316-334.

12. - On the boundary point principle for elliptic equations in the plane, Bull. Amer. Math. Soc. (1967) (to appear.)

13. - Some solutions of elliptic extremal equations in the plane, Le Mate matiche (Catania) (to appear.)

14. M. H. Protter and H. F. Weinberger, Maximum principles in differential equations, Prentice-Hall, Inc., Englewood Cliffs, N. J., 1967.

15. C. Pucci, Un problema variazionale per $i$ coefficienti di equazioni differenziali di tipo ellittico, Ann. Scuola Norm. Sup. Pisa 16 (1962), 159-172.

16. — Operatori ellittici estremanti, Ann. Mat. Pura Appl. 72 (1966), 141-170.

17. - Proprieta di massimo e minimo delle soluzioni di equazioni a derivate parziali del secondo ordine di tipo ellittico e parabolico, Rend. Accad. Naz. Lincei (8) 23 (1957); 24 (1958).

18. R. Vyborny, On properties of solutions of some boundary value problems for equations of parabolic type, Dokl. Akad. Nauk SSSR 117 (1957), 563-565.

Received August 14, 1968. This research was supported in part by Grant AF-AFOSR 1122-67.

University of CAlifornia, Riverside 


\section{PACIFIC JOURNAL OF MATHEMATICS}

\section{EDITORS}

H. ROYDEN
Stanford University
Stanford, California

\section{R. R. PHELPS}

University of Washington

Seattle, Washington 98105
J. DUGUNDJI

Department of Mathematics

University of Southern California

Los Angeles, California 90007

\section{RICHARD ARENS}

University of California

Los Angeles, California 90024

\section{ASSOCIATE EDITORS}

E. F. BeCKenbaCh
B. H. NEUMANN

F. WOLF
K. YoshidA

\section{SUPPORTING INSTITUTIONS}

\author{
UNIVERSITY OF BRITISH COLUMBIA \\ CALIFORNIA INSTITUTE OF TECHNOLOGY \\ UNIVERSITY OF CALIFORNIA \\ MONTANA STATE UNIVERSITY \\ UNIVERSITY OF NEVADA \\ NEW MEXICO STATE UNIVERSITY \\ OREGON STATE UNIVERSITY \\ UNIVERSITY OF OREGON \\ OSAKA UNIVERSITY \\ UNIVERSITY OF SOUTHERN CALIFORNIA
}

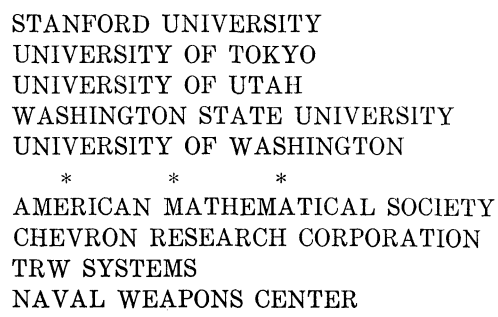

The Supporting Institutions listed above contribute to the cost of publication of this Journal, but they are not owners or publishers and have no responsibility for its content or policies.

Mathematical papers intended for publication in the Pacific Journal of Mathematics should be in typed form or offset-reproduced, double spaced with large margins. Underline Greek letters in red, German in green, and script in blue. The first paragraph or two must be capable of being used separately as a synopsis of the entire paper. It should not contain references to the bibliography. Manuscripts, in duplicate if possible, may be sent to any one of the four editors. Please classify according to the scheme of Math. Rev. 36, 1539-1546. All other communications to the editors should be addressed to the managing editor, Richard Arens, University of California, Los Angeles, California, 90024.

50 reprints are provided free for each article; additional copies may be obtained at cost in multiples of 50 .

The Pacific Journal of Mathematics is published monthly. Effective with Volume 16 the price per volume (3 numbers) is $\$ 8.00$; single issues, $\$ 3.00$. Special price for current issues to individual faculty members of supporting institutions and to individual members of the American Mathematical Society: $\$ 4.00$ per volume; single issues $\$ 1.50$. Back numbers are available.

Subscriptions, orders for back numbers, and changes of address should be sent to Pacific Journal of Mathematics, 103 Highland Boulevard, Berkeley, California, 94708.

PUBLISHED BY PACIFIC JOURNAL OF MATHEMATICS, A NON-PROFIT CORPORATION

Printed at Kokusai Bunken Insatsusha (International Academic Printing Co., Ltd.), 7-17, Fujimi 2-chome, Chiyoda-ku, Tokyo, Japan. 


\section{Pacific Journal of Mathematics \\ Vol. 29, No. $2 \quad$ June, 1969}

Bruce Langworthy Chalmers, On boundary behavior of the Bergman kernel function and related domain functionals ................... 243

William Eugene Coppage, Peirce decomposition in simple Lie-admissible power-associative rings .............................. 251

Edwin Duda, Compactness of mappings...................... 259

Earl F. Ecklund Jr., On prime divisors of the binomial coefficient......... 267

Don E. Edmondson, A modular topological lattice ............... 271

Phillip Alan Griffith, A note on a theorem of Hill ................... 279

Marcel Herzog, On finite groups with independent cyclic Sylow

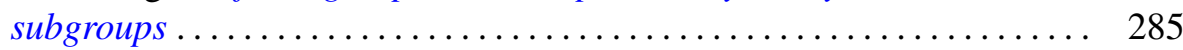

James A. Huckaba, Extensions of pseudo-valuations................. 295

S. A. Huq, Semivarieties and subfunctors of the identity functor ........ 303

I. Martin (Irving) Isaacs and Donald Steven Passman, Finite groups with small character degrees and large prime divisors. II ............ 311

Carl Kallina, A Green's function approach to perturbations of periodic

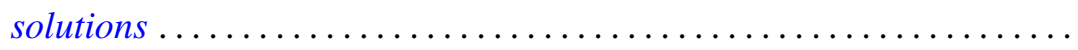

$\mathrm{Al}$ (Allen Frederick) Kelley, Jr., Analytic two-dimensional subcenter manifolds for systems with an integral ....................

Alistair H. Lachlan, Initial segments of one-one degrees ............ 351

Marion-Josephine Lim, Rank k Grassmann products ............. 367

Raymond J. McGivney and William Henry Ruckle, Multiplier algebras of

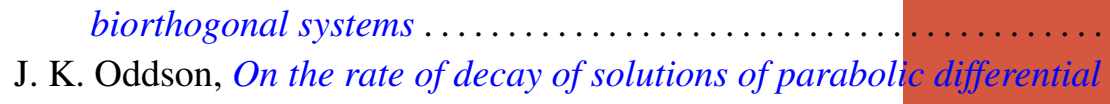

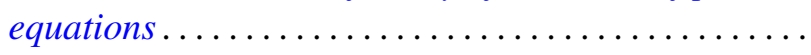

Helmut R. Salzmann, Geometries on surfaces ........... .

Annemarie Schlette, Artinian, almost abelian groups and their groups of automorphisms ............................

Edgar Lee Stout, Additional results on modules over polydisc algebras ...

Lajos Tamássy, A characteristic property of the sphere . .

Mark Lawrence Teply, Some aspects of Goldie's torsion theory. ...

Freddie Eugene Tidmore, Extremal structure of star-shaped sets ...

461

Leon Jarome Weill, Unconditional and shrinking bases in locally convex spaces... 